\title{
Indicação de histerectomia em pacientes acometidas com câncer cervical
}

\author{
Indication of hysterectomy in patients affected with cervical cancer \\ Indicación de histerectomía en pacientes afectados de cáncer cervical
}

Recebido: 30/11/2021 | Revisado: 07/12/2021 | Aceito: 08/12/2021 | Publicado: 16/12/2021

\author{
Eduardo Grasso Júnior \\ ORCID: https://orcid.org/0000-0002-2113-3035 \\ Centro Universitário Uninorte, Brasil \\ E-mail: eb.grassojr@gmail.com \\ Camila da Silva Vieira Amorim \\ ORCID: https://orcid.org/0000-0003-2810-2044 \\ Centro Universitário Uninorte, Brasil \\ E-mail: cajosetti@gmail.com \\ Ruth Silva Lima da Costa \\ ORCID: https://orcid.org/0000-0003-1890-086X \\ Centro Universitário Uninorte, Brasil \\ E-mail: ruttylyma@gmail.com
}

\begin{abstract}
Resumo
A histerectomia é o ato da retirada cirúrgica do útero, realizada por via vaginal, abdominal e abdominal laparoscópica. A decisão pela via de remoção do útero é sugestiva de vários fatores predisponentes e dentre eles o câncer de colo uterino. $\mathrm{O}$ presente estudo objetivo analisar as indicações de histerectomia em pacientes acometidas pelo câncer cervical. Trata-se de uma revisão integrativa da literatura publicada entre 2015 a 2021, realizada nas bases de dados da Medical Literature Analysis Retrievel System Online (MEDLINE), Scientific Eletronic Library Online (SCIELO) e Literatura Latino-americana e do Caribe em Ciências da Saúde (LILACS). Os resultados evidenciaram que a histerectomia é um método de escolha quando o cervical encontra-se avançado ou quando os outros métodos de tratamento não obtiveram o sucesso esperado. Ainda há controvérsias na literatura referente ao momento exato para a realização do procedimento, tendo em vista que alguns autores defendem a idéia de que o procedimento seja realizado nos estágios iniciais da doença. Apesar das controvérsias, a histerectomia tem sido o procedimento adotado para o tratamento do câncer cervical, e tem apresentado resultados satisfatórios quanto ao estadiamento da neoplasia, no entanto os profissionais de saúde necessitam avaliar as possíveis repercussões nas mulheres após a perda do órgão e oferecer o suporte adequado a elas.
\end{abstract}

Palavras-chave: Câncer de colo uterino; Histerectomia; Medicina Preventiva.

\begin{abstract}
Hysterectomy is the act of surgical removal of the uterus, performed by vaginal, abdominal and laparoscopic abdominal routes. The decision to use the uterus removal route is suggestive of several predisposing factors, including cervical cancer. The present study aims to analyze the indications for hysterectomy in patients affected by cervical cancer. This is an integrative literature review published between 2015 and 2021, performed in the Medical Literature Analysis Retrievel System Online (MEDLINE), Scientific Electronic Library Online (SCIELO) and Latin American and Caribbean Health Sciences databases (LILACS). The results showed that hysterectomy is the method of choice when the cervical is advanced or when other treatment methods have not had the expected success. There is still controversy in the literature regarding the exact time to perform the procedure, considering that some authors support the idea that the procedure should be performed in the early stages of the disease. Despite the controversies, hysterectomy has been the procedure adopted for the treatment of cervical cancer, and has shown satisfactory results regarding the staging of the neoplasm. However, health professionals need to assess the possible repercussions on women after organ loss and offer the adequate support for them.
\end{abstract}

Keywords: Cervical cancer; Hysterectomy; Preventive medicine.

\section{Resumen}

La histerectomía es el acto de extirpación quirúrgica del útero, que se realiza por vía vaginal, abdominal y abdominal laparoscópica. La decisión de utilizar la ruta de extirpación del útero sugiere varios factores predisponentes, incluido el cáncer de cuello uterino. El presente estudio tiene como objetivo analizar las indicaciones de la histerectomía en pacientes afectadas por cáncer de cuello uterino. Se trata de una revisión integradora de la literatura publicada entre 2015 y 2021, realizada en el Medical Literature Analysis Retrievel System Online (MEDLINE), Scientific Electronic Library Online (SCIELO) y bases de datos de Ciencias de la Salud de América Latina y el Caribe (LILACS). Los resultados mostraron que la histerectomía es el método de elección cuando el cuello uterino está avanzado o cuando 
otros métodos de tratamiento no han tenido el éxito esperado. Aún existen controversias en la literatura sobre el momento exacto para realizar el procedimiento, considerando que algunos autores apoyan la idea de que el procedimiento debe realizarse en las primeras etapas de la enfermedad. A pesar de las controversias, la histerectomía ha sido el procedimiento adoptado para el tratamiento del cáncer cervicouterino y ha mostrado resultados satisfactorios en la estadificación de la neoplasia, sin embargo, los profesionales de la salud deben evaluar las posibles repercusiones en las mujeres tras la pérdida de órganos y ofrecer el apoyo adecuado para ellos.

Palabras clave: Cáncer de cuello uterino; Histerectomia; Medicina Preventiva.

\section{Introdução}

A histerectomia é o ato da retirada cirúrgica do útero, que pode ser realizada pelas vias vaginal, abdominal e abdominal laparoscópica e de acordo com empresa brasileira de serviços hospitalares (EBSERH), esse procedimento cirúrgico ocupa o segundo lugar entre as cirurgias no sistema público de saúde do Brasil e em mulheres de idade fértil é precedido apenas por parto cirúrgico (Empresa Brasileira de serviços Hospitalares, 2017).

Dados apontam que nos Estados Unidos da América (EUA), somente no ano de 2003 foram realizadas 602.457 histerectomias, das quais $90 \%$ foram realizadas em decorrência de miomas, tumores benignos e endometriose (Costa, 2015).

De acordo com os dados do Ministério da Saúde, através de dados do Departamento de Informação do Sistema Único de Saúde (DATASUS), no período de janeiro a setembro de 2021, foram realizadas 49.045 histerectomias na rede pública de saúde do Brasil, sendo que desse total 21.811 foram histerectomias totais, atualmente ela se configura como a segunda cirurgia ginecológica mais realizada no país, ficando atrás apenas das cesarianas (Brasil, 2021; Silva et al., 2010)

Dados históricos revelam que a primeira histerectomia foi realizada na Alemanha, no ano de 1815, por prolapso urogenital. Com o decorrer do século XIX, o procedimento cirúrgico da retirada do útero pela via vaginal ganhou notável marco na Europa, principalmente na Alemanha e na França, por se tratar de um procedimento que tinha menores riscos de infecções pós-operatórias, em comparação à laparotomia (Murta et al., 2000).

Atualmente, a decisão pela via de remoção do útero dependente de vários fatores predisponentes, que englobam o consentimento da paciente em relação à perda da fertilidade, a técnica e experiência do cirurgião, o local de realização do procedimento e as circunstâncias clínicas das pacientes, e entre as suas mais variadas indicações encontram-se o sangramento uterino anormal, os prolapsos uterinos, os miomas, o câncer benigno, as lesões pré malignas e a adenomiose, assim como, a endometriose (Falcão \& Carvalho,2017).

Mediante a indicação do procedimento como umas das formas de combate ao câncer de colo uterino, principalmente após as falhas de tratamentos anteriores, ressalta-se que esse é o segundo tipo de câncer mais recorrente no Brasil e no mundo, ocorrendo em mulheres na faixa etária entre 40 e 49 anos. Ele configura-se como de fácil rastreio e que cuja morbimortalidade tende a diminuir em $80 \%$ caso identificado precocemente (Instituto Nacional de Câncer José Alencar Gomes da Silva- INCA, 2016).

Dessa forma, salienta-se que nos últimos anos, a realização de histerectomia ou cirurgia de regaste, em pacientes com câncer de colo uterino sem sucesso em outras formas de tratamento, vem demostrando excelentes resultados e obtendo taxas de sobrevida global em 5 anos superiores a 30\% (Coleman et al., 1994; Chiva Lapuente et al.,2008)

Destarte, mediante a necessidade de haver um maior aprofundamento da temática uma vez que os dados sobre o tratamento cirúrgico do câncer do colo uterino após persistência ou recidiva da doença ainda são escassos na literatura, o presente estudo tem por objetivo analisar as indicações de histerectomia em pacientes acometidas pelo câncer cervical. 


\section{Metodologia}

Trata-se de uma revisão bibliográfica descritiva, do tipo revisão integrativa da literatura, usando artigos científicos indexados em banco de dados secundários. A questão norteadora adotada para este estudo foi qual a relação do câncer do colo uterino e a indicação de cirurgia de histerectomia?

As etapas utilizadas na realização dessa revisão foram: (1) identificação de um problema de saúde pública; (2) formulação de uma questão clínica relevante e específica; (3) busca de evidências científicas a partir dos critérios de inclusão e exclusão; (4) avaliação das evidências disponíveis; (5) extração e análise dos dados; (6) síntese e discussão dos resultados.

A seleção dos estudos foi realizada nas seguintes bases de dados eletrônicas: Medical Literature Analysis and Retrievel System Online (MEDLINE), Scientific Eletronic Library Online (SCIELO) e Literatura Latino-americana e do Caribe em Ciências da Saúde (LILACS) com a adoção de descritores (Mesh) "câncer do colo uterino AND histerectomia AND medicina preventiva".

Como critérios de inclusão, foram adotados estudos originais publicados sobre o tema proposto, no período de janeiro de 2015 a novembro de 2021, sem restrição de idioma ou localização, disponíveis online na íntegra e com abordagem completa do conteúdo. Foram excluídos da amostra artigos de relato de caso e documentos.

Após a leitura da extensão completa dos artigos, também foram excluídos os estudos que não respondiam à pergunta da pesquisa e os estudos duplicados.

As buscas resultaram em 52 publicações. Após a aplicação dos critérios mencionados foram encontrados 10 estudos, em seguida, selecionaram-se todos estes estudos para a leitura na íntegra e análise completa, os quais compõem a amostra final desta revisão. Os artigos encontrados foram analisados conforme a ordem cronológica e os dados foram analisados de forma descritiva.

Os dados extraídos das publicações foram organizados e sintetizados um quadro para simplificar a integração dos achados, de acordo com as seguintes variáveis: identificação do artigo (autor e ano da publicação), título, , características metodológicas do estudo, objetivo e resultados, com a finalidade de proporcionar uma análise comparativa, de maneira que estas viabilizassem a elaboração de considerações sobre o tema em estudo.

Quanto aos aspectos éticos, todas as informações extraídas dos artigos pertencem ao domínio público, e as ideias, conceitos e definições dos autores incluídos na revisão foram respeitados, não sendo, portanto, necessária a aprovação do estudo em comitê de ética em pesquisa.

\section{Resultados}

Ao analisar as dez produções científicas incluídas nesta revisão, constatou-se que foram publicados nos anos de 2015 (2), 2016 (1), 2017 (3), 2019 (1), 2020 (2) e 2021 (1). Não foram encontrados estudos no ano de 2018. Os dados apresentados no Quadro 1 mostram as características dos artigos inseridos no presente estudo. 
Quadro 1. Artigos que compõem o corpus da pesquisa.

\begin{tabular}{|c|c|c|c|c|}
\hline AUTOR/ANO & TÍTULO & $\begin{array}{c}\text { DELINEAMEN } \\
\text { TO } \\
\end{array}$ & OBJETIVO & RESULTADOS \\
\hline $\begin{array}{l}\text { Cardoso, } \\
\text { Camargo \& } \\
\text { Fernandes } \\
\text { (2017) }\end{array}$ & $\begin{array}{l}\text { Perfil de mulheres } \\
\text { submetidas a } \\
\text { histerectomia e } \\
\text { influência da } \\
\text { deambulação na alta } \\
\text { hospitalar }\end{array}$ & $\begin{array}{c}\text { Estudo } \\
\text { transversal }\end{array}$ & $\begin{array}{c}\text { Caracterizar o perfil de } \\
\text { mulheres submetidas a } \\
\text { histerectomia e verificar a } \\
\text { influência da deambulação na } \\
\text { alta hospitalar }\end{array}$ & $\begin{array}{l}\text { A amostra contou com } 65 \text { prontuários, sendo o grupo A } \\
\text { com } 37 \% \text { de histerectomia por diagnóstico de câncer de } \\
\text { colo de útero e no grupo B, } 63 \% \text { das mulheres que } \\
\text { realizaram o procedimento não por câncer, porém } \\
\text { constatou-se que a maioria dos prontuários não } \\
\text { especificou o motivo, mas dentre as indicações, a } \\
\text { endometriose foi o motivo mais encontrado. }\end{array}$ \\
\hline $\begin{array}{l}\text { Zanini, Reis, } \\
\text { Laporte, } \\
\text { Vieira, } \\
\text { Zanella, \& } \\
\text { Machado } \\
\text { (2020) }\end{array}$ & $\begin{array}{l}\text { Análise do manejo } \\
\text { cirúrgico de pacientes } \\
\text { com câncer cervical } \\
\text { recidivado após } \\
\text { radioterapia e } \\
\text { quimioterapia }\end{array}$ & $\begin{array}{l}\text { Estudo de coorte } \\
\text { retrospectivo }\end{array}$ & $\begin{array}{l}\text { Analisar os resultados de } \\
\text { morbidade e sobrevida após } \\
\text { cirurgias curativas e paliativas } \\
\text { em pacientes com câncer } \\
\text { cervical recidivado após } \\
\text { tratamento primário com } \\
\text { radioterapia e quimioterapia. }\end{array}$ & $\begin{array}{l}\text { Foram realizadas histerectomias radicais exenterações } \\
\text { pélvicas com intenção curativa e exenterações pélvicas } \\
\text { paliativas. No grupo curativo, houve complicações } \\
\text { maiores em } 40 \% \text { dos casos, e o tempo mediano de } \\
\text { sobrevida foi } 16 \text { meses. No grupo paliativo, houve } \\
\text { complicações maiores em } 60 \% \text { dos casos, e o tempo } \\
\text { mediano de sobrevida foi } 5 \text { meses. Estadiamento } \\
\text { avançado, sintomas, tamanho do tumor maior que cinco } \\
\text { centímetros e mais de três órgãos envolvidos foram fatores } \\
\text { significativamente associados a cirurgia não curativa. }\end{array}$ \\
\hline $\begin{array}{l}\text { Tsunoda, } \\
\text { Andrade, } \\
\text { Vieira \& Reis } \\
\quad(2015)\end{array}$ & $\begin{array}{l}\text { Laparoscopia no câncer } \\
\text { do colo do útero. Estado } \\
\text { atual e revisão da } \\
\text { literatura }\end{array}$ & $\begin{array}{l}\text { Revisão da } \\
\text { literatura }\end{array}$ & $\begin{array}{l}\text { Descrever as principais } \\
\text { aplicações da laparoscopia no } \\
\text { tratamento e estadiamento do } \\
\text { câncer cervical. }\end{array}$ & $\begin{array}{l}\text { O câncer cervical precoce pode ser tratado de forma } \\
\text { adequada por meio de cirurgia minimamente invasiva, } \\
\text { com provável eficácia oncológica semelhante e redução } \\
\text { aparente da morbidade. Para a preservação da fertilidade, } \\
\text { a laparoscopia oferece estadiamento linfonodal adequado } \\
\text { e tratamento com resultados oncológicos semelhantes à } \\
\text { cirurgia radical em pacientes selecionados. }\end{array}$ \\
\hline $\begin{array}{l}\text { Kokka, } \\
\text { Bryant., } \\
\text { Brockbank, } \\
\text { Powell, \& } \\
\text { Oram, } \\
(2015)\end{array}$ & $\begin{array}{l}\text { Histerectomia com } \\
\text { radioterapia ou } \\
\text { quimioterapia ou ambas } \\
\text { para mulheres com } \\
\text { câncer cervical } \\
\text { localmente avançado }\end{array}$ & $\begin{array}{l}\text { Revisão da } \\
\text { literatura }\end{array}$ & $\begin{array}{l}\text { Determinar se a histerectomia, } \\
\text { além do tratamento padrão } \\
\text { com radiação ou } \\
\text { quimioterapia, ou ambos, em } \\
\text { mulheres com câncer cervical } \\
\text { localmente avançado (estágio } \\
\text { IB2 a III) é segura e eficaz em } \\
\text { comparação com o tratamento } \\
\text { padrão sozinho }\end{array}$ & $\begin{array}{l}\text { A partir dos ensaios clínicos randomizados disponíveis, } \\
\text { encontraram-se evidências insuficientes de que a } \\
\text { histerectomia com radioterapia, com ou sem } \\
\text { quimioterapia, melhora a sobrevida de mulheres com } \\
\text { câncer cervical localmente avançado que são tratadas } \\
\text { apenas com radioterapia ou quimiorradioterapia. }\end{array}$ \\
\hline $\begin{array}{l}\text { Diver, } \\
\text { Hinchcliff, } \\
\text { Gockley, } \\
\text { Melamed, } \\
\text { Contrino, } \\
\text { Feldman \& } \\
\text { Growdon, } \\
\text { (2017). }\end{array}$ & $\begin{array}{l}\text { Histerectomia radical } \\
\text { minimamente invasiva } \\
\text { para câncer cervical está } \\
\text { associada a morbidade } \\
\text { reduzida e resultados de } \\
\text { sobrevivência } \\
\text { semelhantes em } \\
\text { comparação com } \\
\text { laparotomia }\end{array}$ & $\begin{array}{l}\text { Estudo de coorte } \\
\text { retrospectivo }\end{array}$ & $\begin{array}{c}\text { Avaliar os resultados de } \\
\text { mulheres com câncer cervical } \\
\text { submetidas à histerectomia } \\
\text { radical (RH) inicial por meio } \\
\text { de uma abordagem de cirurgia } \\
\text { minimamente invasiva (MIS) } \\
\text { ou laparotomia tradicional } \\
\text { (XL) em } 2 \text { grandes instituições } \\
\text { acadêmicas dos EUA para } \\
\text { determinar se o modo de } \\
\text { cirurgia afeta os resultados dos } \\
\text { pacientes. }\end{array}$ & $\begin{array}{l}\text { A Cirurgia não comprometeu os resultados dos pacientes, } \\
\text { incluindo sobrevida geral, taxa de recorrência e frequência } \\
\text { de dissecção ou positividade dos linfonodos pélvicos. A } \\
\text { morbidade foi reduzida no grupo MIS, incluindo menos } \\
\text { transfusões de sangue e menor tempo de internação. }\end{array}$ \\
\hline $\begin{array}{l}\text { Hass, } \\
\text { Eggemann, } \\
\text { Costa, \& } \\
\text { Ignatov, } \\
\text { (2017). }\end{array}$ & $\begin{array}{l}\text { Histerectomia adjuvante } \\
\text { após radio } \\
\text { quimioterapia para } \\
\text { câncer cervical } \\
\text { localmente avançado }\end{array}$ & $\begin{array}{l}\text { Estudo de coorte } \\
\text { prospectivo }\end{array}$ & $\begin{array}{l}\text { Avaliar a morbidade e o } \\
\text { resultado da sobrevida da } \\
\text { histerectomia adjuvante } \\
\text { simples (AH) após } \\
\text { radioterapia e compará-la com } \\
\text { o tratamento padrão. } \\
\end{array}$ & $\begin{array}{l}\text { A histerectomia pode melhorar a sobrevida em pacientes } \\
\text { com doença residual após radioterapia e é caracterizada } \\
\text { por uma baixa taxa de complicações. }\end{array}$ \\
\hline $\begin{array}{l}\text { Oliveira, } \\
\text { Figueiredo, } \\
\text { Dupin, } \\
\text { Salgado, } \\
\text { Barbosa, } \\
\text { Barroso } \\
\text { (2021). }\end{array}$ & $\begin{array}{l}\text { Indicações cirúrgicas } \\
\text { para o tratamento do } \\
\text { câncer de colo uterino } \\
\text { em mulheres jovens }\end{array}$ & $\begin{array}{l}\text { Revisão } \\
\text { sistemática com } \\
\text { metanálise }\end{array}$ & $\begin{array}{l}\text { Investigar as indicações } \\
\text { cirúrgicas após câncer cervical } \\
\text { em mulheres em idade } \\
\text { reprodutiva a fim de informar } \\
\text { sobre os riscos, o prognóstico, } \\
\text { os fatores de risco e os } \\
\text { tratamentos adequados para } \\
\text { melhorar a qualidade de vida } \\
\text { desse grupo de pacientes. }\end{array}$ & $\begin{array}{l}\text { A histerectomia radical é o tratamento cirúrgico padrão } \\
\text { para mulheres com câncer cervical em estágio inicial e a } \\
\text { quimiorradiação é o tratamento para mulheres em estágios } \\
\text { mais avançados. A traquelectomia com linfadenectomia } \\
\text { pélvica é um método alternativo de tratamento para o } \\
\text { câncer cervical precoce sem reduzir a expectativa de vida } \\
\text { e a taxa de recorrência do câncer. Para mulheres que } \\
\text { desejam a preservação da fertilidade, a traquelectomia } \\
\text { radical com remoção do colo do útero e preservação do } \\
\text { útero é uma opção aceita }\end{array}$ \\
\hline $\begin{array}{l}\text { Naumann } \\
\text { (2020). }\end{array}$ & $\begin{array}{l}\text { Ainda é razoável } \\
\text { oferecer histerectomia } \\
\text { minimamente invasiva - } \\
\text { MIS? sim. }\end{array}$ & $\begin{array}{l}\text { Revisão da } \\
\text { literatura }\end{array}$ & $\begin{array}{c}\text { Laparoscopia versus } \\
\text { laparotomia para o tratamento } \\
\text { do câncer cervical em estágio } \\
\text { inicial }\end{array}$ & $\begin{array}{l}\text { A histerectomia radical é o método preferido de } \\
\text { tratamento para o câncer cervical precoce, especialmente } \\
\text { em mulheres mais jovens. }\end{array}$ \\
\hline $\begin{array}{l}\text { Paniago, } \\
\text { (2019). }\end{array}$ & $\begin{array}{l}\text { Avaliação dos métodos } \\
\text { diagnósticos e } \\
\text { terapêuticos de lesões } \\
\text { precursoras de câncer e } \\
\text { câncer do colo uterino } \\
\text { em serviço de referência }\end{array}$ & $\begin{array}{l}\text { Estudo } \\
\text { transversal }\end{array}$ & $\begin{array}{l}\text { Avaliar os aspectos } \\
\text { diagnósticos e terapêuticos das } \\
\text { lesões precursoras do câncer } \\
\text { de colo de útero em um } \\
\text { serviço de referência em } \\
\text { patologia cervical, localizado }\end{array}$ & $\begin{array}{l}\text { A taxa de sobretratamento observada neste estudo foi de } \\
16,3 \% \text { das excisões e } 35,7 \% \text { das histerectomias } \\
\text { evidenciando que a histerectomia é o método de escolha } \\
\text { para o tratamento do câncer cervical. }\end{array}$ \\
\hline
\end{tabular}




\begin{tabular}{|c|c|c|c|c|}
\hline & $\begin{array}{l}\text { secundária de Mato } \\
\text { Grosso do Sul }\end{array}$ & & no HRMS & \\
\hline $\begin{array}{c}\text { Darin, Di } \\
\text { Guilmi, } \\
\text { Verdura, } \\
\text { Sardi, \& Maya } \\
\text { (2016) }\end{array}$ & $\begin{array}{l}\text { Histerectomia radical } \\
\text { laparoscópica: um novo } \\
\text { padrão no tratamento do } \\
\text { câncer cervical? }\end{array}$ & $\begin{array}{l}\text { Estudo } \\
\text { retrospectivo, }\end{array}$ & $\begin{array}{c}\text { Analisar os resultados da } \\
\text { histerectomia radical realizada } \\
\text { por laparoscopia e compará- } \\
\text { los com um grupo controle de } \\
\text { cirurgias abertas. }\end{array}$ & $\begin{array}{l}\text { A histerectomia radical laparoscópica mostrou } \\
\text { recuperação mais rápida com menor tempo de internação. } \\
\text { Os tempos cirúrgicos e complicações não mostraram } \\
\text { diferenças significativas. }\end{array}$ \\
\hline
\end{tabular}

Fonte: Dados da Pesquisa (2021).

\section{Discussão}

Conforme observado no quadro 1 , o estudo em questão demonstrou que a histerectomia é um método de escolha quando o câncer do colo uterino se encontra avançado ou quando os outros métodos de tratamento não obtiveram o sucesso esperado. Ainda há controvérsias na literatura referente ao momento exato para a realização do procedimento, tendo em vista que alguns autores defendem a idéia de que o procedimento seja realizado nos estágios iniciais da doença (Tsunoda et al., 2015; Oliveira et al., 2021; Naumann 2020).

Ressalta-se que atualmente algumas patologias que acometem a pelve da mulher, e dentre elas destaca-se o câncer cervical, se configuraram como um sério problema de saúde pública, pois além das questões associadas a patologia em si, destacam-se ainda o fato de que esse órgão está relacionado a feminilidade, o que pode gerar complicações de ordem também psicoemocionais (Silva et al., 2016; Teixeira \& Batista, 2016).

Dentre os procedimentos de escolha para o tratamento do câncer cervical, encontra-se a indicação da histerectomia, que consiste na retirada total o útero, sendo que ela é a segunda maior causa de cirurgias realizadas em mulheres no Brasil pelo Sistema Único de Saúde (SUS) (Silva et al., 2010), além do que para fins de tratamento da doença, é o que tem apresentado melhor resposta a prevenção da doença invasiva e cura (Conde et al., 2018; Camargo \& Fernandes 2017; Hass et al., 2017; Darin et al., 2016).

A histerectomia pode ser classificada em total, subtotal e radical. No total é realizada a remoção do útero e colo cervical; no subtotal ocorre à retirada do útero com a preservação do colo uterino e na radical, remove-se todo o útero, incluindo o colo uterino e outros órgãos adjacentes como tubas uterinas e ovários (Marques \& Mota, 2010)

Nesse sentido, as mulheres que realizam o procedimento, seja ele total, subtotal ou radical, necessitarão de suporte físico e emocional por uma equipe multiprofissional, no sentido de aumentar sua autoestima e amenizar as aflições a respeito do procedimento cirúrgico, que como já mencionado anteriormente, essa mudança poder levar a crença da perda de feminilidade, por ser abrupta na vida das mulheres acometidas (Silva et al., 2010; Teixeira \& Batista, 2016; Barbosa et al., 2018).

Destarte, dados da literatura, evidenciam que a histerectomia total é o procedimento de escolha para o tratamento do câncer cervical em mulheres jovens, uma vez que permite a preservação da função ovariana, bem como é considerado o padrão para o tratamento, apresentando bons resultados na sobrevida e com grandes chances de redução de complicações (Chuang et al, 2016; Suarez-Candena, 2018).

De acordo com os achados de Nagura et al. (2014), a histerectomia radical, continua sendo o procedimento mais indicado quando o tamanho do tumor é maior que $3 \mathrm{~mm}$ de um carcinoma de células escamosas, corroborando com os achados de Diver et al. (2017), Oliveira et al. (2021), Naumann (2020) e Darin et al. (2016).

Independentemente do tipo de técnica cirúrgica utilizada, o impacto do procedimento, sobre a função sexual, não está ainda bem definido na literatura, pois segundo dados, a prevalência de problemas relacionados a disfunção sexual, variam de acordo com a diferenças metodológicas da cirurgia (Illiano et al., 2016).

Atualmente outro método cirúrgico, chamado de histerectomia robótica, vem sendo bastante utilizando e recomendando pois apresenta menor, tempo cirúrgico, menos perda sanguínea, maior sobrevida e menos complicações em 
menos de seis semanas após o procedimento (Yim et al., 2014), sendo que a histerectomia radical pode atualmente ser realizada por via minimamente invasiva, tanto por laparoscopia ou também por robótica. (Fialho \& Esteves (2017).

Dessa forma, esses novos métodos, vem evidenciando resultados promissores contra o controle e invasão do câncer cervical, no entanto mais pesquisas devem ser realizadas com vistas a identificar qual deles é o mais adequado para o combate à doença (Suarez-Cadena, 2018).

Do ponto de vista preventivo, reafirma-se a necessidade do rastreio inicial do câncer do colo uterino, como medidas de promoção e prevenção da saúde, através da realização de exames ginecológicos de frequência nas mulheres em idade reprodutiva, e realização do diagnóstico e tratamento precoce, afim de evitar a necessidade da realização de cirurgias invasivas, proporcionando assim mais qualidade de vida à mulher (Freitas et al., 2016).

\section{Considerações Finais}

Mediante aos achados, evidencia-se que indicação da histerectomia como parte do tratamento do câncer cervical, tem sido amplamente utilizada. Após o estadiamento com exatidão, com seu devido suporte multiprofissional, a histerectomia é a melhor resolução definitiva para o grupo de mulheres alvo.

No entanto, sendo o ato cirúrgico muito utilizado na população feminina para fins de controle do câncer cervical, seja ele nos momentos iniciais da doença, ou quando as outras tentativas de tratamento não foram eficazes, há a necessidade de mais aprofundamento no que se diz respeito ao rastreamento precoce desse tipo de câncer, para assim evitar cirurgias irreversíveis desnecessárias, ao qual podem afetar diretamente na rotina da mulher, sua autoestima, seu desejo de reprodução, possíveis complicações pós cirúrgicas, além reafirmar uma indicação cirúrgica correta.

Novos estudos frente a essa temática são necessários com o intuito de dar visibilidade ao tema em questão e subsidiar profissionais de saúde com informações atuais baseado nas evidencias cientificas mais recentes sobre o tema em questão.

\section{Referências}

Barbosa, A. R. D. S., dos Santos, A. N., \& Rodrigues, T. S. (2018). Experiência de mulheres que realizaram histerectomia: revisão integrativa. Revista Uningá, 55(2), 227-241.

Brasil. Datasus - Departamento de informática do SUS. Ministério da Saúde, 2021. Sistema de Informações hospitalares SUS (SIH/SUS). http://tabnet.datasus.gov.br/cgi/tabcgi.exe?sih/cnv/qiuf.def.

Cardoso, B. C., Camargo, C. R., \& Fernandes, I. (2017). Perfilde Mulheres Submetidas a Histerectomia e Influência da Deambulação na Alta Hospitalar. Revista Pleiade, 11(21), 17-24.

Chiva, L. M., Lapuente, F., González-Cortijo, L., González-Martín, A., Rojo, A., García, J. F., \& Carballo, N. (2008). Surgical treatment of recurrent cervical cancer: state of the art and new achievements. Gynecologic oncology, 110(3), S60-S66.

Coleman, R. L., Keeney, E. D., Freedman, R. S., Burke, T. W., Eifel, P. J., \& Rutledge, F. N. (1994). Radical hysterectomy for recurrent carcinoma of the uterine cervix after radiotherapy. Gynecologic oncology, 55(1), 29-35.

Costa, J.R.C.C (2015). Tipos e vias de abordagem cirúrgica da histerectomia e sua relação com lesão do sistema urinário. Rev ActaObs-tetGinecolPort, São Paulo, 6(1) 2-4.

Darin, C., Di Guilmi, J., Verdura, R., Sardi, J., \& Maya, G. A. (2016). Histerectomía radical laparoscópica: un nuevo estándar en el tratamiento del cáncer de cuello uterino. Fronteras en Medicina, 11(1), 13-16.

Diver, E., Hinchcliff, E., Gockley, A., Melamed, A., Contrino, L., Feldman, S., \& Growdon, W. (2017). Minimally invasive radical hysterectomy for cervical cancer is associated with reduced morbidity and similar survival outcomes compared with laparotomy. Journal of minimally invasive gynecology, 24(3), 402406.

Ebserh. Empresa Brasileira de serviços hospitalares (2017). Protocolo clínicos e diretrizes terapêuticas, Escolha de via da histerectomia. Maternidade Escola Assis Chateaubriand, 9,169-171.

Falcão, F., \& Carvalho, G. (2017). Cirurgia por via vaginal. Revista Catarinense de Medicina, 23(8), 1-30.

Fialho, F. A., \& Esteves, D. C. (2017). Câncer de colo do útero: O mérito da Prevenção. Rev Conex eletrônica, 14(1), 142-153. 
Conde, C. R., Lemos, T. M. R., \& Ferreira, M. D. L. D. S. M. (2018). Características sociodemográficas, individuales y de programación de las mujeres con cáncer cervical. Enfermería Global, 17(49), 348-380

Freitas, C. B., Gomes, N. P., Campos, L. M., Estrela, F. M., Cordeiro, K. C. C., \& dos Santos, R. M. (2016). Complicações pós-cirúrgicas da histerectomia: revisão integrativa. Revista Baiana de Enfermagem30,(2).

Hass, P., Eggemann, H., Costa, S. D., \& Ignatov, A. (2017). Adjuvant hysterectomy after radiochemotherapy for locally advanced cervical cancer. Strahlentherapie und Onkologie, 193(12), 1048-1055.

Illiano E, Giannitsas K, Costantini E. (2016) Hysterectomy and sexuality. First Published. Disponível em: www.avidscience.com.

Suarez-Cadena, F. C. (2018). Terapéutica del cáncer de cuello uterino, una revisión de la literatura. MedUNAB, 21(1), 100-114.

Instituto Nacional de Câncer José Alencar Gomes da Silva- INCA (2016). Diretrizes brasileiras para o rastreamento do câncer do colo do útero /. Coordenação de Prevenção e Vigilância. Divisão de Detecção Precoce e Apoio à Organização de Rede. (2a ed.).

Kokka, F., Bryant, A., Brockbank, E., Powell, M., \& Oram, D. (2015). Hysterectomy with radiotherapy or chemotherapy or both for women with locally advanced cervical cancer. Cochrane Database of Systematic Reviews, (4).

Marques, J. P., \& Mota, F. (2010). Cirurgia uterina por via abdominal. Oliveira CF. Manual de ginecologia. Permanyer Portugal, 447-79

Murta, E. F. C., Reis, J. D. D., Abrão, J. M., \& Miziara, J. M. (2000). Histerectomias: estudo retrospectivo de 554 casos. Revista do Colégio Brasileiro de Cirurgiões, 27, 307-311.

Nagura, M., Koshiyama, M., Matsumura, N., Kido, A., Baba, T., Abiko, K., \& Konishi, I. (2014). Clinical approaches to treating papillary squamous cell carcinoma of the uterine cervix. BMC câncer, 14(1), 1-7.

Naumann, R. W. (2020). ¿Sigue siendo razonable ofrecer histerectomía MIS? Sí. Contemporaray Ob GYN. Enero 20. 65

Oliveira J. P., et al. (2021). Indicações cirúrgicas para o tratamento do câncer de colo uterino em mulheres jovens. Brazilian Journal of Surgery and Clinical Research-BJSCR, 33(3), 94-103.

Paniago, M. L., (2019). Avaliação dos métodos diagnósticos e terapêuticos de lesões precursoras de câncer e câncer do colo uterino em serviço de referência secundária de Mato Grosso do Sul. 86 f. Dissertação (Mestrado Profissional) FIOCRUZ.

Silva, C. D. M. C., Santos, I. M. M. D., \& Vargens, O. M. D. C. (2010). A repercussão da histerectomia na vida de mulheres em idade reprodutiva. Escola Anna Nery, 14, 76-82.

Teixeira, M. R., \& Batista, E. C. (2017). Vivências cotidianas da mulher histerectomizada: narrativas e contextos. Revista Enfermagem e Saúde ColetivaREVESC, 1(2), 91-17.

Tsunoda, A. T., Andrade, C. E. M. D. C., Vieira, M. A., \& Reis, R. D. (2015). Laparoscopy in uterine cervical cancer. Current state and literature review. Revista do Colégio Brasileiro de Cirurgiões, 42, 345-351.

Yim, G. W., Kim, S. W., Nam, E. J., Kim, S., Kim, H. J., \& Kim, Y. T. (2014). Surgical outcomes of robotic radical hysterectomy using three robotic arms versus conventional multiport laparoscopy in patients with cervical cancer. Yonsei medical journal, 55(5), 1222-1230.

Zanini, L. A. G., Reis, R. J., Laporte, G. A., Vieira, S. C., Zanella, J. D. F., \& Machado, G. M. (2020). Análise do manejo cirúrgico de pacientes com câncer cervical recidivado após radioterapia e quimioterapia. Revista do Colégio Brasileiro de Cirurgiões, 47. 